\title{
On the Turing complexity of learning finite families of algebraic structures ${ }^{\star}$
}

\author{
Nikolay Bazhenov ${ }^{1[0000-0002-5834-2770]}$ and \\ Luca San Mauro 2 [0000-0002-3156-6870] \\ 1 Sobolev Institute of Mathematics, 4 Acad. Koptyug Ave., Novosibirsk, \\ 630090, Russia \\ bazhenov@math.nsc.ru \\ 2 Institute of Discrete Mathematics and Geometry, Vienna University of Technology \\ luca.sanmauro@gmail.com
}

\begin{abstract}
In previous work, we have combined computable structure theory and algorithmic learning theory to study which families of algebraic structures are learnable in the limit (up to isomorphism). In this paper, we measure the computational power that is needed to learn finite families of structures. In particular, we prove that, if a family of structures is both finite and learnable, then any oracle which computes the Halting set is able to achieve such a learning. On the other hand, we construct a pair of structures which is learnable but no computable learner can learn it.
\end{abstract}

Keywords: Inductive inference · Algorithmic learning theory · Computable structures · Infinitary logic · Turing degrees.

\section{Introduction}

Algorithmic learning theory, introduced by Gold 12 in the 1960's, comprises different formal models for the inductive inference. Broadly construed, this research program deals with the question of how a learner, provided with more and more data about some environment, is eventually able to achieve systematic knowledge about it (see [14 for an introduction to this area). Most work in algorithmic learning theory concerns either learning of recursive functions [25] or learning of formal languages [17. These paradigms model the data to be learned as an unstructured flow. Yet, researchers have been also considering (although less systematically) the learning of data embodied with a structural content, often focusing on special classes of algebraic structures: e.g., in a series of papers, Stephan and his collaborators considered commutative rings [24], trees [20], vector spaces [13], and matroids [10].

\footnotetext{
* Bazhenov was supported by the Mathematical Center in Akademgorodok under agreement No. 075-15-2019-1613 with the Ministry of Science and Higher Education of the Russian Federation. San Mauro was supported by the Austrian Science Fund FWF, project M 2461.
} 
A broader pursuit is to develop a framework which can be applied to arbitrary structures. This has been done first by Glymour [11] and later expanded by Martin and Osherson [19. We contributed to this research thread with our own paradigm [815, which is inspired by ideas and techniques from computable structure theory. Intuitively (but see below for formal details), we say that a countably infinite structure $\mathcal{A}$ is learnable if one can eventually guess its isomorphism type by seeing larger and larger (but always finite) substructures of $\mathcal{A}$. As for classical paradigms, the emphasis is not on learning single structures but rather families of structures (the former case being trivial).

In [5], we adopted infinitary logic to obtain a model-theoretic characterization of which families of structures are learnable. Remarkably, there are families of structures which are finite, up to isomorphism, and not learnable; this contrasts with classical paradigms, since, e.g., any finite collection of recursive functions is learnable.

In this paper, we advance the knowledge about the learnability of finite families of structures. Specifically, by relying on Turing complexity we evaluate the computational power that is needed to learn such families. We prove that there is a pair of structures which is learnable but no computable learner can learn it, solving a question left open in [5].

\section{Preliminaries}

We assume that the reader is familiar with the basic notions of classical computability theory; in any case, our terminology and notations are standard and as in [23. In particular, by $\left\{\varphi_{e}\right\}_{e \in \omega},\left\{W_{e}\right\}_{e \in \omega}$, and $\left\{\Phi_{e}^{X}\right\}_{e \in \omega}$ we denote a uniformly computable list of, respectively, all partial computable functions, all computably enumerable (c.e.) sets, and all Turing operators with oracle $X$.

Computable structures. A signature $L$ lists all function symbols and relation symbols which characterize an algebraic structure. In this paper, we consider only relational signatures, i.e., signatures with no function symbols. Furthermore, all our structures have domain the set $\omega$ of the natural numbers. We say that two structures are copies of each other if they are isomorphic. In computable structure theory, one measures the complexity of an $L$-structure $\mathcal{A}$ by identifying $\mathcal{A}$ with its atomic diagram, i.e., the collection of atomic formulas which are true of $\mathcal{A}$. Up to a suitable Gödel numbering of $L$-formulas, the atomic diagram of $\mathcal{A}$ may be regarded as a subset of $\omega$ : this provides a natural way of assigning to each structure a Turing degree $\mathbf{d}$, representing its algorithmic complexity. Any computable structure $\mathcal{A}$ in a relational signature can be presented as an increasing union of its finite substructures

$$
\mathcal{A} \uparrow_{0} \subseteq \mathcal{A} \uparrow_{1} \subseteq \ldots \subseteq \mathcal{A} \uparrow_{i} \subseteq \ldots,
$$

where $\mathcal{A}\left\lceil_{n}\right.$ denotes the restriction of $\mathcal{A}$ to the domain $\{0,1, \ldots, n\}$ and $\mathcal{A}=$ $\cup \mathcal{A} \uparrow_{i}$. For more background about computable structures, see [317]. 
Infinitary formulas. To assess the model-theoretic complexity of countable structures, it is common to work in the infinitary logic $\mathcal{L}_{\omega_{1} \omega}$, which allows to take the conjunctions or disjunctions of infinite sets of formulas. In particular, infinitary $\Sigma_{n}$ formulas are defined as follows,

$-\Sigma_{0}^{\inf }$ and $\Pi_{0}^{\mathrm{inf}}$ formulas are quantifier-free first-order formulas.

- A $\sum_{n+1}^{\inf }$ formula $\psi(\bar{x})$ is a countably infinite disjunction

$$
\bigvee_{i \in I} \exists \bar{y}_{i} \xi_{i}\left(\bar{x}, \bar{y}_{i}\right)
$$

where each $\xi_{i}$ is a $\Pi_{n}^{\text {inf }}$ formula.

- A $\Pi_{n+1}^{\mathrm{inf}}$ formula $\psi(\bar{x})$ is a countably infinite conjunction

$$
\mathbb{M}_{i \in I} \forall \bar{y}_{i} \xi_{i}\left(\bar{x}, \bar{y}_{i}\right)
$$

where each $\xi_{i}$ is a $\Sigma_{n}^{\text {inf }}$ formula.

Next, computable infinitary $\Sigma_{n}$ formulas (or $\Sigma_{n}^{c}$ formulas, for short) are defined in the same way as above, but requiring infinite conjunctions and disjunctions to range over c.e. sets of (computable) formulas. Finally, computable infinitary formulas can be relativized to an arbitrary oracle $X$ : the class of $X$-computable infinitary $\Sigma_{n}$ formulas is denoted by $\Sigma_{n}^{c}(X)$. For more background about infinitary formulas, see [18].

\subsection{Our framework (for finite families)}

We shall now revisit $\mathbf{I n f} \mathbf{E x}_{\mathbf{x}}$, the learning paradigm presented in [5. Here, the exposition is somehow simplified by the fact that we will focus only on finite families of structures: this allows us to ignore how a given family is enumerated, which was a crucial source of complexity in [5]. For current purposes, it is in fact enough to assume that any structure $\mathcal{A}$ gives rise to a corresponding conjecture $\ulcorner\mathcal{A}\urcorner$, to be understood as conveying the piece of information "this is $\mathcal{A}$ ".

Suppose that $\mathbf{P}$ is the learning problem associated to a finite family $\mathfrak{K}$ of (non-isomorphic) computable structures. The ingredients of our framework may be specified as follows. For $\mathbf{P}$,

- The learning domain (LD) is the collection of all copies of the structures from $\mathfrak{K}$. That is,

$$
\operatorname{LD}(\mathfrak{K}):=\bigcup_{\mathcal{A} \in \mathfrak{K}}\{\mathcal{S}: \mathcal{S} \cong \mathcal{A}\} .
$$

- The hypothesis space (HS) contains, for each $\mathcal{A} \in \mathfrak{K}$, a formal symbol $\ulcorner\mathcal{A}\urcorner$ (these symbols serve as conjectures about the isomorphism type of the observed structure) and a question mark symbol. That is,

$$
\operatorname{HS}(\mathfrak{K}):=\{\ulcorner\mathcal{A}\urcorner: \mathcal{A} \in \mathfrak{K}\} \cup\{?\} .
$$


- A learner $M$ sees, by stages, all positive and negative data about any given structure in the learning domain and is required to output conjectures. This is formalized by saying that $M$ is a function

$$
\text { from }\left\{\mathcal{S} \uparrow_{n}: \mathcal{S} \in L D(\mathfrak{K})\right\} \text { to } \operatorname{HS}(\mathfrak{K}) .
$$

- The learning is successful if, for each structure $\mathcal{S} \in \mathfrak{K}$, the learner eventually stabilizes to a correct conjecture about its isomorphism type. That is,

$$
\lim _{n \rightarrow \infty} M\left(\mathcal{S} \uparrow_{n}\right)=\ulcorner\mathcal{A}\urcorner \text { if and only if } \mathcal{S} \text { is a copy of } \mathcal{A} \text {. }
$$

We say that $\mathfrak{K}$ is learnable, if some learner $M$ successfully learns $\mathfrak{K}$.

Remark 2.1. The interested reader is referred to 5 for motivating examples and a detailed discussion about our framework. Note that in that paper, inspired by an established notation in algorithmic learning theory, we named our paradigm

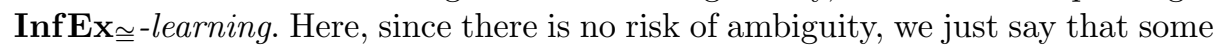
family $\mathfrak{K}$ is learnable.

In [5], we showed that asking if a family (possibly infinite) is learnable is the same as asking whether the structures from $\mathfrak{K}$ can be distinguished by $\Sigma_{2}^{\inf }$ formulas. The next theorem is an immediate consequence of Theorem 3.1 and Corollary 4.1 of $[5]$.

Theorem 2.1 (Bazhenov, Fokina, San Mauro). Let $\mathfrak{K}$ be a finite family of pairwise nonisomorphic structures $\left\{\mathcal{A}_{0}, \ldots, \mathcal{A}_{n}\right\}$. Then,

(a) $\mathfrak{K}$ is learnable if and only if there are $\Sigma_{2}^{\text {inf }}$ formulas $\phi_{0}, \ldots, \phi_{n}$ such that

$$
\mathcal{A}_{i} \models \phi_{j} \Leftrightarrow i=j .
$$

(b) $\mathfrak{K}$ is learnable via an $X$-computable learner if and only if there are $\Sigma_{2}^{c}(X)$ formulas $\psi_{0}, \ldots, \psi_{n}$ such that

$$
\mathcal{A}_{i} \models \psi_{j} \Leftrightarrow i=j
$$

\section{An upper bound to the learners' complexity}

According to our framework, a learner $M$ can be a function of any complexity. So, it is natural to ask how learnability is affected if we restrict our focus to learners of some bounded Turing complexity. More precisely, in this paper we want to understand, for a learnable family $\mathfrak{K}$, how powerful an oracle should be to achieve such learning. The next theorem states that, if $\mathfrak{K}$ is finite, then $\mathbf{0}^{\prime}$ suffices. Similar problems for classical learning paradigms have been intensively studied: e.g., Kummer and Stephan [16] showed, the whole of class of c.e. languages is learnable from informant relative to oracle $A$ if and only if the Halting problem is Turing reducible to $A$ (for more results about learning with oracles, see [122[16]). 
Theorem 3.1. Let $\mathfrak{K}$ be a finite family of computable structures. If $\mathfrak{K}$ is learnable, then it is learnable by a $\mathbf{0}^{\prime}$-computable learner.

Proof. Suppose that $\mathfrak{K}$ is equal to $\left\{\mathcal{A}_{0}, \ldots, \mathcal{A}_{n}\right\}$. By item $(a)$ of Theorem 2.1. there are $\Sigma_{2}^{\text {inf }}$ sentences $\phi_{0}, \ldots, \phi_{n}$ such that for all $i, j \leq n$,

$$
\mathcal{A}_{j} \models \phi_{i} \Leftrightarrow j=i .
$$

We show that one can replace formulas $\phi_{i}$ with $\Sigma_{2}^{c}\left(\mathbf{0}^{\prime}\right)$ sentences $\psi_{i}$, while preserving Eq. (1). Then item (b) of Theorem 2.1 will imply that the class $\mathfrak{K}$ is learnable by a $\mathbf{0}^{\prime}$-computable learner. We describe the construction of $\psi_{0}$ - the remaining $\psi_{i}$ can be recovered in a similar way.

Suppose that $\phi_{0}$ is equal to

$$
\bigvee_{i \in I} \exists \bar{x}_{i} \xi_{i}\left(\bar{x}_{i}\right)
$$

where every $\xi_{i}$ is a $\Pi_{1}^{\text {inf }}$ formula. Without loss of generality, one may assume that $\mathcal{A}_{0} \models \exists \bar{x}_{0} \xi_{0}\left(\bar{x}_{0}\right)$. By Eq. (1), we have

$$
\mathcal{A}_{\ell} \not \models \exists \bar{x}_{0} \xi_{0}\left(\bar{x}_{0}\right) \text { for all } \ell \neq 0 \text {. }
$$

Suppose

$$
\xi_{0}\left(\bar{x}_{0}\right)=\mathbb{M}_{j \in J} \forall \bar{y}_{j} \theta_{j}\left(\bar{x}_{0}, \bar{y}_{j}\right)
$$

where every $\theta_{j}$ is quantifier-free.

Choose a tuple $\bar{c}$ from $\mathcal{A}_{0}$ such that $\mathcal{A}_{0} \models \xi_{0}(\bar{c})$. Consider a set of formulas

$$
\operatorname{type}_{\forall}(\bar{c})=\left\{\chi\left(\bar{x}_{0}\right): \chi \text { is a finitary } \forall \text {-formula, } \mathcal{A}_{0} \models \chi(\bar{c})\right\} .
$$

Since $\mathcal{A}_{0}$ is a computable structure, the set $\operatorname{type}_{\forall}(\bar{c})$ is co-c.e. Furthermore, $\left\{\forall \bar{y}_{j} \theta_{j}\left(\bar{x}_{0}, \bar{y}_{j}\right): j \in J\right\} \subseteq \operatorname{type}_{\forall}(\bar{c})$.

Consider a $\Sigma_{2}^{c}\left(\mathbf{0}^{\prime}\right)$ sentence

$$
\psi_{0}:=\exists \bar{x}_{0} \bigwedge_{\chi \in \text { type }(\bar{c})} \chi\left(\bar{x}_{0}\right)
$$

By employing Eq. (2), it is easy to show that $\mathcal{A}_{0}=\psi_{0}$ and $\mathcal{A}_{\ell} \not \models \psi_{0}$ for every $\ell>0$. In other words, the formula $\psi_{0}$ satisfies the desired properties. We deduce that $\mathfrak{K}$ is learnable by a $\mathbf{0}^{\prime}$-computable learner.

The following question, which naturally originates from the theorem above, is left open.

Question 3.1. Is there an intermediate degree $\mathbf{0}<\mathbf{d}<\mathbf{0}^{\prime}$ such that, if a finite family $\mathfrak{K}$ is learnable, then it is learnable by a $\mathbf{d}$-computable learner? 


\section{A lower bound to the learners' complexity}

Having shown that an oracle as weak as $\mathbf{0}^{\prime}$ is able to retrieve the learning process for any finite family which is learnable, one may ask whether, for finite families, learnability even coincides with computable learnability. In this section, we show that this is not the case, by constructing a pair of structures which, although learnable, cannot be learned by any computable learner. This answers the question left open in [5, p. 17]. Our construction is based on a family of structures built by Alaev [2].

Theorem 4.1. There is a pair $\mathfrak{K}$ of computable structures such that $\mathfrak{K}$ is learnable, but not learnable by a computable learner.

Proof. For the sake of exposition, the proof is split into several subsections.

\section{Preliminaries of the construction}

We treat trees as undirected graphs. Consider a relational signature $L_{0}:=$ $\left\{R^{2}, Q^{1}, U^{1}\right\}$. We define an auxiliary computable $L_{0}$-structure $\mathcal{V}$ as follows.

- The domain of $\mathcal{V}$ is equal to

$$
\{r, c\} \cup\left\{a_{i}, b_{i}: i \in \omega\right\} \cup\left\{d_{i, j}: i \in \omega, j \leq i\right\} .
$$

$-Q^{\mathcal{V}}=\{c\}$ and $U^{\mathcal{V}}=\{r\} \cup\left\{a_{i}, b_{i}: i \in \omega\right\}$.

- The structure $\left(\operatorname{dom}(\mathcal{V}), R^{\mathcal{V}}\right)$ is an undirected graph, which contains the following edges:

- $\left(r, a_{i}\right)$ and $\left(a_{i}, b_{i}\right)$ for all $i \in \omega$;

- $\left(a_{i}, d_{i, 0}\right),\left(d_{i, 0}, d_{i, 1}\right),\left(d_{i, 1}, d_{i, 2}\right), \ldots,\left(d_{i, i-1}, d_{i, i}\right),\left(d_{i, i}, c\right)$.

The informal idea behind the structure $\mathcal{V}$ is as follows. If we consider its substructure $\mathcal{S}$ on the domain $U^{\mathcal{V}}$, then $\mathcal{S}$ is a tree with root $r$ and infinitely many branches of size 2 .

For a given $i \in \omega$, the elements $d_{i, j}, j \leq i$, serve to distinguish $a_{i}$ in a firstorder way. Let $\theta_{i}\left(x, y_{0}, \ldots, y_{i}, z\right)$ be a quantifier-free formula, which is built as the conjunction of the following facts:

- all elements $x, y_{0}, \ldots, y_{i}, z$ are pairwise distinct;

$-U(x)$ and $Q(z)$

$-\neg U\left(y_{j}\right)$ for all $j \leq i$;

- the sequence $x, y_{0}, \ldots, y_{i}, z$ forms a chain with respect to the graph relation $R$.

Then we have:

$$
\mathcal{V} \models\left(x=a_{i}\right) \leftrightarrow \exists y_{0} \ldots \exists y_{i} \exists z \theta_{i}(x, \bar{y}, z)
$$

We use the structure $\mathcal{V}$ to code c.e. sets. For a c.e. set $W$, by $\mathcal{T}[W]$ we denote the substructure of $\mathcal{V}$ on the c.e. domain

$$
\{r, c\} \cup\left\{a_{i}: i \in \omega\right\} \cup\left\{d_{i, j}: i \in \omega, j \leq i\right\} \cup\left\{b_{i}: i \in W\right\} .
$$




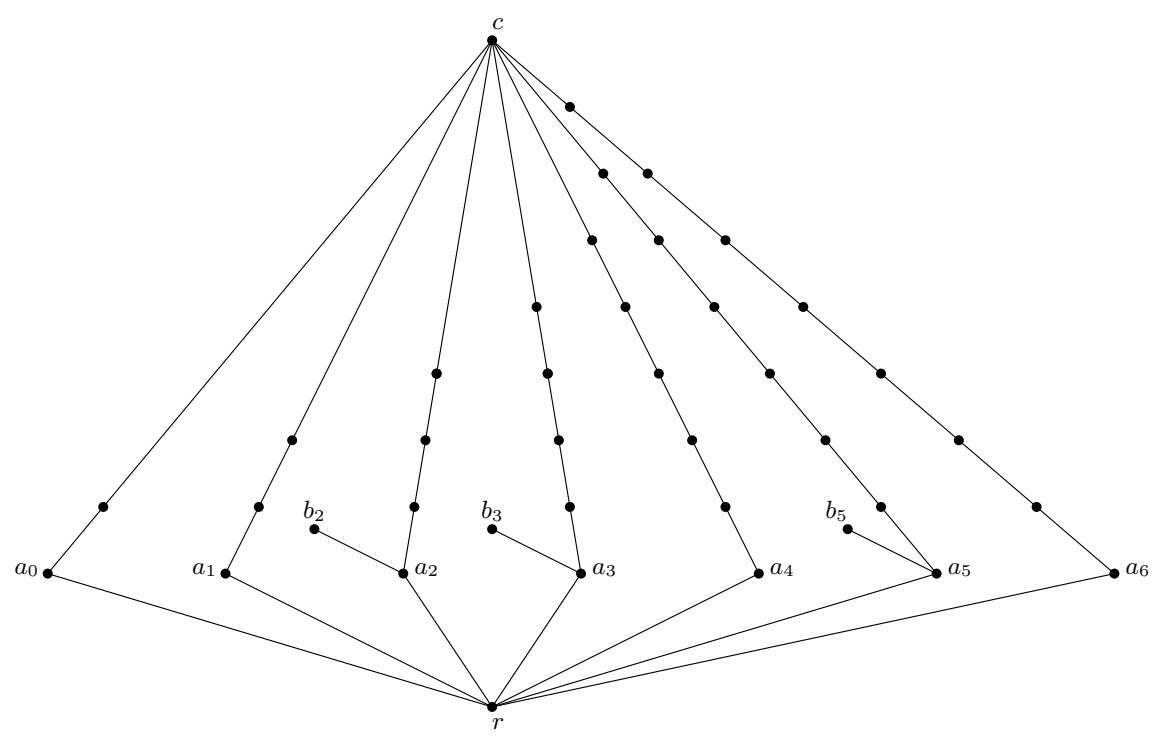

Fig. 1. A fragment of $\mathcal{T}[P]$, where $P$ is the set of all primes.

See Fig. 1 for an example of this encoding. Without loss of generality, one may assume that the structure $\mathcal{T}[W]$ is computable. Furthermore, given an index $e \in \omega$, one can effectively find a computable index of the structure $\mathcal{T}\left[W_{e}\right]$.

We will use the following technical lemmas:

Lemma 4.1 (folklore). The set $\left\{e \in \omega: W_{e} \subseteq \emptyset^{\prime}\right\}$ is $\Pi_{2}^{0}$-complete.

Lemma 4.2 (see Lemma 5 of [2]). Let $\mathcal{I}$ be a family, which consists of all c.e. sets $W$ such that $\emptyset^{\prime} \subseteq W$ and $\operatorname{card}\left(W \backslash \emptyset^{\prime}\right)=1$. The family $\mathcal{I}$ has the following properties.

1. I is uniformly enumerable, i.e. there is a computable function $h(x)$ such that the family $\left\{W_{h(e)}: e \in \omega\right\}$ equals $\mathcal{I}$. Note that in general, the sequence $\left(W_{h(e)}\right)_{e \in \omega}$ allows repetitions.

2. There is a computable function $f_{\mathcal{I}}(x)$ so that for every $x, W_{f_{\mathcal{I}}(x)}=\emptyset^{\prime} \cup\{x\}$. In particular, for all $e \in \omega$ :

(2.a) If $W_{e} \subseteq \emptyset^{\prime}$, then $W_{f_{\mathcal{I}}(e)}=\emptyset^{\prime}$.

(2.b) If $W_{e} \nsubseteq \emptyset^{\prime}$, then $W_{f_{\mathcal{I}}(e)} \in \mathcal{I}$.

\section{Construction}

Choose an arbitrary set $X \in \Delta_{3}^{0} \backslash \Delta_{2}^{0}$.

Fix two binary $\Pi_{2}^{0}$ predicates $P_{+}$and $P_{-}$such that for any $k \in \omega$,

$$
\begin{aligned}
& k \in X \Leftrightarrow \exists x P_{+}(k, x) ; \\
& k \notin X \Leftrightarrow \exists x P_{-}(k, x) .
\end{aligned}
$$


Let $\circ \in\{+,-\}$. By Lemma 4.1, there is a computable function $g_{\circ}(k, x)$ such that $P_{\circ}(k, x)$ is equivalent to the condition $W_{g_{\circ}(k, x)} \subseteq \emptyset^{\prime}$. Hence, by Lemma 4.2 , we have:

- If $P_{\circ}(k, x)$ is true, then $W_{f_{\mathcal{I}}\left(g_{\circ}(k, x)\right)}=\emptyset^{\prime}$.

- If $P_{\circ}(k, x)$ is false, then $W_{f_{\mathcal{I}}\left(g_{\circ}(k, x)\right)} \in \mathcal{I}$.

Consider a signature $L:=L_{0} \cup\left\{T^{1} ; a\right\}$, where $a$ is a constant symbol. For a number $k \in \omega$, we define a computable $L$-structure $\mathcal{C}_{k}$ as follows. If it is not specified otherwise, we assume that a freshly added element $x$ of $\mathcal{C}_{k}$ satisfies $\neg T(x)$.

(A) Choose a fresh element $r^{k}$ as the root of the tree. Put $a^{\mathcal{C}_{k}}:=r^{k}$.

(B) For each set $W \in \mathcal{I}$, append to $r^{k}$ infinitely many copies $\mathcal{D}_{\ell}, \ell \in \omega$, of the structure $\mathcal{T}[W]$. For each of $\mathcal{D}_{2 m}, m \in \omega$, we set $\mathcal{C}_{k}=T(q)$, where $q$ is the root of $\mathcal{D}_{2 m}$.

(C) For each $\circ \in\{+,-\}$ and each $m \in \omega$, append to $r^{k}$ infinitely many copies $\mathcal{E}_{\ell}, \ell \in \omega$, of the structure $\mathcal{T}\left[W_{f_{\mathcal{I}}\left(g_{\circ}(k, m)\right)}\right]$. Let $q$ be the root of $\mathcal{E}_{\ell}$. If o equals + , then set $\mathcal{C}_{k}=T(q)$.

Since the family $\mathcal{I}$ is uniformly enumerable, it is not hard to show that the structures $\mathcal{C}_{k}$ are uniformly computable.

Let $\mathcal{C}_{\text {base }}$ be the $L$-structure obtained by employing only the steps (A) and (B) described above. From (4), it is not hard to establish the following:

1. If $k \in X$, then $\mathcal{C}_{k}$ is isomorphic to the structure $\mathcal{C}^{+}$, which is constructed by appending to $\mathcal{C}_{\text {base }}$ infinitely many copies of $\mathcal{T}\left[\emptyset^{\prime}\right]$ with their roots satisfying the predicate $T$.

2. If $k \notin X$, then $\mathcal{C}_{k}$ is isomorphic to the structure $\mathcal{C}^{-}$, which is obtained by appending to $\mathcal{C}_{\text {base }}$ infinitely many copies of $\mathcal{T}\left[\emptyset^{\prime}\right]$ with their roots satisfying the formula $\neg T(x)$.

\section{Verification}

Consider class $\mathfrak{K}$, containing the isomorphism types of the structures $\mathcal{C}^{+}$and $\mathcal{C}^{-}$. We prove that the class $\mathfrak{K}$ satisfies our theorem.

We define the following $\Sigma_{2}^{\text {inf }}$-sentences: for $\circ \in\{+,-\}$,

$$
\begin{aligned}
& \xi_{\circ}:=\exists x\left[R(a, x) \& T^{\circ}(x) \&\right. \\
& \mathbb{M}_{i \in \overline{\bar{b}^{\prime}}} \forall \widehat{x} \forall y_{0} \ldots \forall y_{i} \forall z\left[R(x, \widehat{x}) \& \theta_{i}\left(\widehat{x}, y_{0}, \ldots, y_{i}, z\right) \rightarrow\right. \\
&\neg \exists v(R(\widehat{x}, v) \& U(v) \& v \neq x)]],
\end{aligned}
$$

where $\theta_{i}$ are formulas from Eq. (3), and

$$
T^{\circ}(x)= \begin{cases}T(x), & \text { if } \circ=+, \\ \neg T(x), & \text { if } \circ=-.\end{cases}
$$


The structure $\mathcal{C}^{+}$satisfies the sentence $\xi_{+}$: the desired element $x$ can be chosen as the root of some appended tree, which is isomorphic to $\mathcal{T}\left[\emptyset^{\prime}\right]$. On the other hand, one can show that $\mathcal{C}^{-} \not \models \xi_{+}$.

Indeed, towards a contradiction, assume that some $x_{-} \in \mathcal{C}^{-}$has the described properties. Then $x_{-}$is the root of some appended tree, which is isomorphic to $\mathcal{T}[W]$ for a set $W$ belonging to the class $\mathcal{I}$. Recall that there is a unique element $\ell \in W \backslash \emptyset^{\prime}$. Furthermore, there is a unique tuple $\widehat{x}, y_{0}, \ldots, y_{\ell}, z$ from $\mathcal{C}^{-}$with the property $R\left(x_{-}, \widehat{x}\right) \& \theta_{\ell}\left(\widehat{x}, y_{0}, \ldots, y_{\ell}, z\right)$. Roughly speaking, in the appended tree, we have $\widehat{x}=a_{\ell}, y_{j}=d_{\ell, j}$, and $z=c^{\text {of this tree }}$. Since $\ell \in W$, the element $b_{\ell}$ belongs to the tree, therefore,

$$
\mathcal{C}^{-} \models \exists v\left(R(\widehat{x}, v) \& U(v) \& v \neq x_{-}\right),
$$

which gives a contradiction.

In a similar way, one shows that $\mathcal{C}^{-} \models \xi_{-}$and $\mathcal{C}^{+} \not \xi_{-}$. By Theorem 2.1 $(a)$, we deduce that our class $\mathfrak{K}$ is learnable.

Now, towards a contradiction, assume that the class $\mathfrak{K}$ is learnable by a computable learner $M$. Without loss of generality, one may assume the following: if a copy of the structure $\mathcal{C}^{\circ}$ (where $\circ \in\{+,-\}$ ) is given as input, then in the limit, $M$ will output the symbol o.

Consider the computable sequence $\left(\mathcal{C}_{k}\right)_{k \in \omega}$ built in the construction. Then we have the following:

- If $k \in X$, then $\mathcal{C}_{k} \cong \mathcal{C}^{+}$. Thus, given the data about $\mathcal{C}_{k}$, the learner $M$ outputs + in the limit.

- If $k \notin X$, then $\mathcal{C}_{k} \cong \mathcal{C}^{-}$. Given $\mathcal{C}_{k}$, the limit output of $M$ is equal to - .

Since the learner $M$ is computable, we deduce that $X$ is a $\Delta_{2}^{0}$ set, which contradicts the choice of our $X$. Therefore, $\mathfrak{K}$ is not learnable in a computable fashion. Theorem 4.1 is proved.

\section{Conclusion}

Let us conclude by briefly mentioning two ways of extending the above research.

First, one may say that an oracle $X$ is low for learning structures if any family $\mathfrak{K}$ which is learnable by an $X$-computable learner can already be learned computably. A number of lowness notions have been investigated in computability theory (e.g., an oracle can be low for isomorphism [9], low for bi-embeddability [4, low for randomness [21, etc.). Yet, the study of oracles which do not supplement the learning power of $\mathbf{0}$ (called trivial) is also an important tradition in algorithmic learning theory: Slaman and Solovay 22 proved that, in the case of learning recursive functions, noncomputable trivial oracles coincide with the 1 -generic sets below $\emptyset^{\prime}$. So, one could try to characterize the class of oracles that are low for learning structures.

The second research direction that we want to suggest builds on the following observation: the results of this paper are all based on the fact that one could 
effectively recover (a copy of) a given structure $\mathcal{A}$ from knowing the conjecture $\ulcorner\mathcal{A}\urcorner$. What if one drops this assumption? For instance, suppose that a learner is allowed to output as conjectures any index of the observed structure (according to some background enumeration of the computable structures). We would like to know whether the addition of multiple indices for a given structure extends the capabilities of computable learners (as is in the case of behaviourally correct learning, see [6[15]).

\section{References}

1. Adleman, L.M., Blum, M.: Inductive inference and unsolvability. Journal of Symbolic Logic 56(3), 891-900 (1991). https://doi.org/10.2307/2275058

2. Alaev, P.E.: Constructive and non-constructive infinite formulas in computable structures. Algebra and Logic 42(4), 219-231 (2003). https://doi.org/10.1023/A:1025053225562

3. Ash, C.J., Knight, J.F.: Computable Structures and the Hyperarithmetical Hierarchy, Studies in Logic and the Foundations of Mathematics, vol. 144. Elsevier Science B.V., Amsterdam (2000)

4. Bazhenov, N., Fokina, E., Rossegger, D., San Mauro, L.: Degrees of bi-embeddable categoricity. Computability 10(1), 1-16 (2021). https://doi.org/10.3233/COM190289

5. Bazhenov, N., Fokina, E., San Mauro, L.: Learning families of algebraic structures from informant. Information and Computation 275, 104590 (2020). https://doi.org/10.1016/j.ic.2020.104590

6. Case, J., Smith, C.: Comparison of identification criteria for machine inductive inference. Theoretical Computer Science 25(2), 193-220 (1983). https://doi.org/10.1016/0304-3975(83)90061-0

7. Ershov, Y.L., Goncharov, S.S.: Constructive models. Kluwer Academic/Plenum Publishers, New York (2000)

8. Fokina, E., Kötzing, T., San Mauro, L.: Limit learning equivalence structures. In: Garivier, A., Kale, S. (eds.) Proceedings of the 30th International Conference on Algorithmic Learning Theory. Proceedings of Machine Learning Research, vol. 98, pp. 383-403. PMLR, Chicago, Illinois (22-24 Mar 2019)

9. Franklin, J.N.Y., Solomon, R.: Degrees that are low for isomorphism. Computability 3(2), 73-89 (2014). https://doi.org/10.3233/COM-140027

10. Gao, Z., Stephan, F., Wu, G., Yamamoto, A.: Learning families of closed sets in matroids. In: Dinneen, M.J., Khoussainov, B., Nies, A. (eds.) Computation, Physics and Beyond - International Workshop on Theoretical Computer Science, WTCS 2012. Lecture Notes in Computer Science, vol. 7160, pp. 120-139. Springer, Berlin (2012). https://doi.org/10.1007/978-3-642-27654-5_10

11. Glymour, C.: Inductive inference in the limit. Erkenntnis 22, 23-31 (1985). https://doi.org/10.1007/BF00269958

12. Gold, E.M.: Language identification in the limit. Information and Control 10(5), 447-474 (1967). https://doi.org/10.1016/S0019-9958(67)91165-5

13. Harizanov, V.S., Stephan, F.: On the learnability of vector spaces. Journal of Computer and System Sciences 73(1), 109-122 (2007). https://doi.org/10.1016/j.jcss.2006.09.001

14. Jain, S., Osherson, D., Royer, J.S., Sharma, A.: Systems that learn: An introduction to learning theory. MIT Press (1999) 
15. Jain, S., Sharma, A.: On the non-existence of maximal inference degrees for language identification. Information Processing Letters 47(2), 81-88 (1993). https://doi.org/10.1016/0020-0190(93)90229-3

16. Kummer, M., Stephan, F.: On the structure of degrees of inferability. Journal of Computer and System Sciences 52(2), 214-238 (1996). https://doi.org/10.1006/jcss.1996.0018

17. Lange, S., Zeugmann, T., Zilles, S.: Learning indexed families of recursive languages from positive data: A survey. Theoretical Computer Science 397(1-3), 194-232 (2008). https://doi.org/10.1016/j.tcs.2008.02.030

18. Marker, D.: Lectures on infinitary model theory, Lecture Notes in Logic, vol. 46. Cambridge University Press, Cambridge (2016). https://doi.org/10.1017/CBO9781316855560

19. Martin, E., Osherson, D.: Elements of scientific inquiry. MIT Press (1998)

20. Merkle, W., Stephan, F.: Trees and learning. Journal of Computer and System Sciences 68(1), 134-156 (2004). https://doi.org/10.1016/j.jcss.2003.08.001

21. Nies, A.: Lowness properties and randomness. Advances in Mathematics 197(1), 274-305 (2005). https://doi.org/10.1016/j.aim.2004.10.006

22. Slaman, T.A., Solovay, R.: When oracles do not help. In: Warmuth, M.K., Valiant, L.G. (eds.) Proceedings of the Fourth Annual Workshop on Computational Learning Theory. pp. 379-383. Morgan Kaufmann Publishers Inc., San Francisco (1991)

23. Soare, R.I.: Turing Computability. Theory and Applications. Springer, Berlin (2016)

24. Stephan, F., Ventsov, Y.: Learning algebraic structures from text. Theoretical Computer Science 268(2), 221-273 (2001). https://doi.org/10.1016/S03043975(00)00272-3

25. Zeugmann, T., Zilles, S.: Learning recursive functions: A survey. Theoretical Computer Science 397(1-3), 4-56 (2008). https://doi.org/10.1016/j.tcs.2008.02.021 